Théologiques

Théologiques

\title{
Entre persuasion et adhésion
}

\section{La mission française au XVII ${ }^{\mathrm{e}}$ siècle}

\section{Dominique Deslandres}

Volume 13, numéro 1, printemps 2005

Croire et croyances

URI : https://id.erudit.org/iderudit/012527ar

DOI : https://doi.org/10.7202/012527ar

Aller au sommaire du numéro

\section{Éditeur(s)}

Faculté de théologie et de sciences des religions, Université de Montréal

\section{ISSN}

1188-7109 (imprimé)

1492-1413 (numérique)

Découvrir la revue

Citer cet article

Deslandres, D. (2005). Entre persuasion et adhésion : la mission française au $\mathrm{XVII}^{\mathrm{e}}$ siècle. Théologiques, 13(1), 95-117. https://doi.org/10.7202/012527ar
Résumé de l'article

La rencontre des croyances franco-amérindiennes du xvii e siècle doit être replacée dans le contexte plus global des missions de cette époque. On voit alors se développer simultanément les missions à l'intérieur de la France, auprès des catholiques tièdes et des protestants, et les missions à l'extérieur de la métropole, auprès entre autres des Amérindiens de Nouvelle-France. Or, l'analyse révèle que, dans son ensemble, la mission française au xvii ${ }^{\mathrm{e}}$ siècle fut loin d'être un lieu d'innovation. L'altérité, en effet, a eu un impact quasi nul sur les mentalités et les méthodes missionnaires. D’une part, les missionnés étaient perçus essentiellement comme des « ignorants » de « ce qui est nécessaire à salut ", dont il serait facile de circonvenir les superstitions ; d'autre part, c'est l'ensemble des stratégies missionnaires des différents ordres et des congrégations à l'intérieur de la France qui se trouva "importé " en Nouvelle-France. Dans ce processus, la rencontre de l' Autre se fait à sens unique, les missionnaires étant là pour donner et non pour recevoir des missionnés quelque chose qui pourrait les remettre en question ou susciter leur adhésion à la religion de l’ Autre .
Tous droits réservés @ Faculté de théologie et de sciences des religions, Université de Montréal, 2006
Ce document est protégé par la loi sur le droit d'auteur. L'utilisation des services d'Érudit (y compris la reproduction) est assujettie à sa politique d'utilisation que vous pouvez consulter en ligne.

https://apropos.erudit.org/fr/usagers/politique-dutilisation/ 


\title{
Entre persuasion et adhésion
}

La mission française au XVII ${ }^{\mathrm{e}}$ siècle

\author{
Dominique Deslandres \\ Département d'histoire \\ Université de Montréal
}

Il y a quelques années, le film Black Robe $e^{1}$ relatait l'histoire du père Laforgue, un jésuite français qui, au début de la colonie française, se trouve en mission chez les Hurons et en vient à douter de sa mission et de sa foi. Le récit s'achève sur le sentiment que le missionnaire français va peut-être même se convertir à la foi de l'Autre. Une telle représentation est tout à fait dans la mentalité hollywoodienne qui projette volontiers les mentalités d'aujourd'hui sur celles du passé. Et j'entends souvent mes étudiants supposer qu'il est possible de trouver une attitude d'ouverture, de tolérance et d'adhésion chez les agents convertisseurs français du XVII ${ }^{\mathrm{e}}$ siècle. Je dois les détromper et leur rappeler que le missionnaire catholique français de cette époque n'est pas un homme qui doute - il sait; il ne doute pas, il est convaincu d'avoir raison - d'autre part, si les mœurs de l'Amérindien peuvent parfois l'intéresser, si les pratiques de ces «païens sous un vernis chrétien » qui habitent la France et qu'il cherche à ramener dans le giron de l'Église catholique, peuvent souvent l'intriguer, d'une manière générale, la foi de l'Autre lui est inadmissible, incorrecte, impossible ${ }^{2}$. En aucun cas, elle ne saurait susciter son adhésion.

1. Film réalisé en 1991 par l'Australien Bruce Beresford à partir du roman Black Robe (1985) du Canadien Brian Moore.

2. La plupart du temps, en effet, dans les relations qu'ils font de leurs missions, les missionnaires catholiques du XVII ${ }^{e}$ siècle décrivent l'Autre au pire comme un être sans vraie religion, au mieux comme un être pratiquant une forme de christianisme abâtardie par l'ignorance, la superstition, l'hérésie. À ce sujet, voir Seumois 1952, 300301; Delacroix 1957, 15 ; Daniel-Rops 1966, 233-243 ; Rogier et al. 1968, 18-19; Delumeau et Cottret 1996, 147-148. Voir plus particulièrement : Farriss 1984; Duverger 1987, 191-193; Gruzinski 1988, 367; Clendinnen 1987, 229-245; Clendinnen 1990, 105-141; MacCormack 1991 ; Zupanov 1991 ; Deslandres 1997b, 618-664 ; Griffiths et Cervantes 1999. 
J'aimerais réfléchir ici sur les modalités et les termes de cette rencontre des croyances, qui s'est produite au XvII ${ }^{\mathrm{e}}$ siècle, quand les Français ont débarqué dans la vallée laurentienne et ont été confrontés aux religions autochtones. Or, comme je l'ai montré dans Croire et faire croire. Les missions françaises an $17^{e}$ siècle (2003), cette confrontation ne peut être comprise que si elle est replacée dans le contexte plus global des missions de cette époque, un contexte qui voit se développer simultanément les missions à l'intérieur de la France, auprès des catholiques tièdes et des protestants, et les missions à l'extérieur de la métropole, auprès principalement des Amérindiens de Nouvelle-France. Répandre le christianisme du concile de Trente fonde l'esprit de ces missions (Deslandres 2003).

\section{L'impact quasi nul de l'altérité sur les mentalités et méthodes missionnaires}

Malgré les apparences, malgré ce que longtemps on a pu en dire, la mission française au XVII ${ }^{e}$ siècle fut loin d'être un lieu d'innovation. En effet, pour les missionnaires, formés à l'école du concile de Trente, il ne s'agissait nullement d'innover en matière de dogme ou de pratiques religieuses ni «d'introduire dans une chose établie », ici le catholicisme romain, "quelque chose de nouveau, d'encore inconnu ", comme le suppose, depuis le Xvi siècle, le terme innover ${ }^{3}$.

Le nouveau, l'inconnu, aurait pu être constitué par l'altérité présentée par les peuples amérindiens, une altérité radicale dont l'introduction aurait suscité des changements dans la religion vécue par l'ensemble des fidèles français, mais cela ne s'est pas produit. Le nouveau, l'inconnu, aurait pu être introduit dans les méthodes pour aborder et convertir des peuples qui nous apparaissent, à nous, fort différents des Européens de l'époque, mais cela ne s'est pas produit. Le nouveau, l'inconnu, enfin, aurait pu être vécu, analysé, compris par les missionnaires qui, pour lors, s'en seraient trouvés profondément transformés, changés, mais cela ne s'est pas produit. Tout cet ensemble de réactions hypothétiques ne s'est pas produit dans la rencontre euroamérindienne et cela pour plusieurs raisons qui ont trait autant à la perception de Soi et de l'Autre qu'à la compréhension "intégriste », voire «fondamentaliste» de la religion chrétienne au XVII ${ }^{\mathrm{e}}$ siècle.

3. Le Petit Robert, version cédérom, 1995, sous l'article «Innover ». 


\subsection{La confrontation avec une altérité radicale}

Rappelons que du point de vue sémantique, ce qui est «autre » qualifie ce qui est différent, distinct. Du point de vue philosophique, ce qui est "autre» constitue une catégorie de l'être et de la pensée, qualifiant l'hétérogène, le divers, le multiple (par opposition au même). Du point de vue occidental de l'histoire, fondé par la tradition européocentrique, ce qui est «autre» est tout simplement ce qui n'est pas européen. Et ce qui n'est pas européen frappe de plein fouet l'imaginaire de l'Europe à partir du Xvi ${ }^{\mathrm{e}}$ siècle ${ }^{4}$ (voir O'Gorman 1961; Elliott 1972; Pagden 1982; Todorov 1982 et 1989; Grafton et al. 1992, 28-58, 116; Axtell 1992, 25-74). Cet imaginaire a jusqu'alors intégré les musulmans, ces voisins méditerranéens, compétiteurs commerciaux et militaires, tributaires des Écritures, mais ennemis de la vraie foi. Cet imaginaire a intégré jusqu'aux Chinois et Indiens des Indes, ces barbares extrême-orientaux dont les invraisemblables richesses arrivent à l'Europe par les caravanes et que, selon la tradition, les apôtres auraient déjà christianisés.

Et voilà qu'avec la découverte des Amériques, il faudrait aux Européens penser une altérité radicale; il leur faudrait comprendre que d'autres êtres existent totalement en dehors de la Révélation. Or, cette compréhension, qui peut nous sembler évidente aujourd'hui, va totalement à l'encontre de la mentalité de l'époque et de la tradition qui fait remonter à Adam la création du monde et qui lui confère le titre de père de l'humanité (voir Gliozzi 2000 ; Elliot 1970, 15-16; Elliot 1976, 11-23 ; Cañizares-Esguerra 2002). Et la reconnaissance de l'Autre, pour ce qu'il est, dans sa valeur intrinsèque, va être très, très lente à se construire. Même les esprits les plus favorables vont résister à l'idée d'un Autre, radicalement différent, car cet Autre ne peut, en aucun cas, avoir des origines antébibliques ou carrément hors la Bible. Or, dans son existence même, cet Autre est dérangeant, incongru, déplacé au plus haut titre parce qu'il ne correspond à rien, à aucun modèle connu. Mais si on l'accepte tel quel, c'est toute l'autorité des Écritures qui s'effondre, c'est véritablement la fin du monde. C'est pourquoi il a fallu, aux missionnaires et aux théologiens de l'époque, trouver un moyen de justifier l'existence de l'Autre, incarné par l'Américain. Ils ont louvoyé et se sont efforcés par tous les moyens de récupérer cette humanité carrément différente dans un système familier de pensée; ils ont développé des thèses

4. Pour une récente discussion de ce sujet dans le contexte de la colonisation hollandaise du Nouveau Monde, voir Schmidt 2002. 
comme celle des tribus d'Israël perdues et retrouvées ou encore la thèse de la Révélation prototype, selon laquelle la Révélation aurait été répandue dans tous les coins du monde puis, faute de pasteurs, elle aurait été oubliée par les peuples ou pire encore parodiée en des superstitions ridicules... Nous retrouvons exactement ces considérations dans les efforts des jésuites de Nouvelle-France à retrouver des notions du Déluge dans les religions amérindiennes ${ }^{5}$.

De telles résistances, profondes, pérennes et puissantes, peuvent peutêtre s'expliquer par le fait qu'au début du XvII ${ }^{\mathrm{e}}$ siècle, on commence à peine à s'extraire de la pensée analogique; on est encore loin d'explorer, à l'instar de Descartes, la pensée objective, fondée sur le doute. La majorité des gens continue à juger autrui (le prochain par rapport à soi) à l'intérieur du pareil à soi, hors de tout doute. Ces "Sauvages » américains, après avoir été jugés êtres humains et non plus animaux, doivent être littéralement ramenés à la chrétienté qu'ils ont eu le tort d'oublier, ils doivent revenir, pense-t-on, à la seule religion véritable, la seule pensable: en dehors d'elle, point de salut... Nous sommes donc, au début du XvII ${ }^{\mathrm{e}}$ siècle, dans un contexte précartésien, où le respect des Auctoritates, comme on dit alors, prime sur la connaissance déduite, où le « je crois donc je suis » prime sur le « je pense donc je suis ", où la liberté en matière de religion est littéralement odieuse, où un Clément VIII réagissant à l'édit de Nantes, pouvait s'exclamer: « ... la liberté de conscience à tout un chacun [...] est la pire chose du monde» (cité par Audisio 1996, 383).

\subsection{Tous des ignorants}

Comme je l'ai montré ailleurs par l'étude des Indipetce (ces suppliques que les jésuites envoyaient au général de la compagnie pour solliciter leur départ aux Indes), les missionnaires voient de la même façon négative les catholiques tièdes, les paysans ignorants, les hérétiques français, les païens du MoyenOrient et des Indes, les Turcs et les Sauvages du Canada. Ces missionnés sont alors tous considérés essentiellement comme des «ignorants » de «ce

5. «Vous voyez qu'ils ont quelque tradition du déluge, quoy que meslée de fables, car voicy comme le monde se perdit, à ce qu'ils disent " (Campeau 1979, 434); "Pour le Messou, ils tiennent qu'il a réparé le monde qui s'estoit perdu par le déluge d'eau, d'où appert qu'ils ont quelque tradition de cette grande inondation universelle qui arriva du temps de Noë, mais ils ont remply cette vérité de mille fables impertinentes. » (Campeau 1979, 564 ; voir 1967, 65, 75 ; 1987, 248, 347-348, 563, 565 ; 1996, 834) 
qui est nécessaire à salut » et ils sont jugés sur le même pied d'égalité; voire les réactions qu'ils ont face à la mission sont très exactement prévues par les missionnaires qui s'y préparent en conséquence (voir Deslandres 1997a, 505-538). Aussi, les nouveaux chrétiens de Nouvelle-France sont-ils loués parce qu'ils pratiquent " exactement ", "réglément » comme on dit alors, le christianisme tridentin, ce christianisme que les missionnaires tentent, par tous les moyens, d'implanter en France. À Sillery, écrit-on :

La fréquentation des sacremens, l'avidité qu'ont ces bons néophytes de la parole de Dieu, l'observance qu'ils rendent à ses commandemens, leur diligence pour assister à la saincte messe tous les jours, les chastimens qu'ils commencent d'exercer sur les délinquants, leur zèle pour la deffence et pour l'amplification de la foy sont autant de marques que Jésus-Christ s'affermit dans leur cœur. (B. Vimont, Relation de 1642, dans Campeau 1990, 381)

C'est l'écho de ce qu'on entend en France. Dans le diocèse de Châlons, par exemple, la mission de Jean Eudes:

... produisit en effet des changements étonnants dans tous les états, dans toutes les conditions, et dans l'un et l'autre sexe [...]. On fréquenta davantage les sacrements et l'on y apporta, avec plus de soin, les dispositions requises pour les recevoir avec fruit. La piété prit dans un grand nombre de lieux la place du désordre et du libertinage. On estima davantage la religion, on la respecta, on en aima les pratiques. La régularité fut plus grande dans le clergé et le peuple eut plus de zèle pour suivre l'exemple de ses pasteurs ${ }^{6}$.

6. Jean Eudes, cité dans Berthelot du Chesnay 1967, 179. Voir Bourdoise 1660, 67, 6970, 73 et passim; Descourveaux 1714, 106, 79. Charles de Genève note :

C'est une chose admirable de voir le peuple qui pour l'ordinaire, a tant de peine d'assister aux offices divins ès jours de festes, en ces occasions, quoyque préssé de plusieurs travaux et oppressé de plusieurs charges, venir unanimement et de si bon cœur entendre la parolle de Dieu ès jours ouvriers, quittant tout pour s'exposer aux ardeurs de l'été, avec un si grand concours de diverses parroisses, de lieux si éloignés, nonobstant quelle injure de temps que ce soit, d'un esprit et courage du tout infatigable, pour rendre à Dieu et à son Église ste des fruicts abondants, voire jusques au centième. Il seroit bien difficile à décrire ou racconter les grandes émotions des esprits, les admirables métamorphoses et changemens de vies, combien de rancunes invétérées depuis plusieurs années se terminent en des mutuels ambrassements, en syncères amitiés d'une paix vrayement chrétienne ! Combien de gens d'une vie désespérée qui retournent à leur devoir ! Combien de personnes qui de toutes les semaines entières, à peine seront sorties de l'église pour prendre un peu de réfection! (Charles de Genève 1976, vol. 3, 162-165)

Voir aussi, dans le cas du jésuite Maunoir en Bretagne, Maunoir 1998, 38, 48, 52, 59, 60, 72, 104, 107-108, 48; Boschet 1834, 170, 145, 244-245, 286-287. 
Les récits missionnaires s'extasient ainsi devant le respect, l'obéissance, la ferveur, l'assiduité avec lesquels les missionnés s'acquittent des «saints exercices de la piété », ces dévotions encouragées par le concile de Trente, qui vont de la participation à la messe au signe de la croix, en passant par la récitation du chapelet ou la génuflexion devant le Saint Sacrement. Fréquentation des sacrements, assistance assidue à la messe et aux fêtes, respect des prêtres, des églises, des dévotions tridentines... Sans compter le zèle à se confesser, à se mortifier... Tout y est, dans les relations missionnaires, pour faire la preuve qu'on peut, même au sein des plus ignorants parmi les barbares, transplanter tel quel le christianisme de Trente.

La nouveauté, si elle existe, c'est cette volonté chez les élites d'imposer au plus grand nombre un modèle, devisé à l'origine pour une poignée d'élu(e)s (Delumeau 1981, 93 ; voir 84-85 ; 1978, 393-394 ; 1983, 331-333; 1989), car on n'a pas assez relevé le fait que ce christianisme tridentin qu'on implante à l'intérieur de la France et qu'on exporte outremer au XVII ${ }^{\mathrm{e}}$ siècle se fonde sur le retour à l'esprit et la façon de l'Église primitive. En tous cas, il est clair que pour les missionnaires français de l'époque, il s'agit de suivre l'exemple des apôtres. Ils ne cessent de comparer leurs efforts aux travaux apostoliques des premiers chrétiens. Ainsi Vincent de Paul peut-il écrire: «... tout ce que nous faisons du matin jusqu'au soir ne doit tendre qu'à nous rendre bons chrétiens mais tels qu'étaient ceux de la primitive Église. Nous n'aurons pas fait peu si nous pouvons arriver à leur charité, à leur zèle » (Dodin 1960, 1027 (28 maxime; voir aussi Delumeau 1981, 162-164).

\subsection{Reconnaître, "s'accoustumer"}

Ces apôtres modernes poursuivent les mêmes buts que ceux des premiers disciples du Christ, et, fait marquant, ils « reconnaissent » dans les réactions, les ferveurs et les pénitences que suscitent leurs missions le reflet de la première Église; en Savoie comme à Sillery, en Bretagne comme en Huronie, «il sembloit, à voir ces spectacles, que le temps de la primitive Église fût retourné » (Charles de Genève 1976, vol. 1, 80 ; voir vol. 3, 172, 181, 242). Comme l'écrivit Paul Le Jeune, le supérieur jésuite de la mission canadienne: « Plus cette Eglise naissante a de rapport avec la primitive, plus nous donne[t]-elle d'espérance de luy voir porter des fleurs et des fruicts dignes du 
paradis $^{7} »($ Relation de 1637, dans Campeau 1987, 525). Ce désir ardent de se calquer sur l'Église des premiers apôtres explique pourquoi le nouveau, l'inconnu, n'a pu être introduit dans les méthodes pour aborder et convertir des peuples qui nous apparaissent, à nous, fort différents des Européens de l'époque.

Or, l'analyse comparée des moyens de conversion employés de part et d'autre de l'Atlantique confirme par ailleurs la remarquable continuité de la méthodologie missionnaire utilisée dans les missions de France et de Nouvelle-France. Signalons au passage que l'apprentissage des langues vernaculaires qui nous semble, vu du XxI ${ }^{\mathrm{e}}$ siècle, le summum de l'adaptation et de l'innovation, est un indispensable prérequis aux missionnaires de l'intérieur comme de l'extérieur de la France. En effet, l'attention, d'ailleurs toute tridentine, portée à la langue utilisée dans le pays missionné est un des traits remarquables de l'intégration socioreligieuse de cette époque ${ }^{8}$. Et les problèmes linguistiques, rencontrés chez les Amérindiens par les missionnaires sont ceux qu'ils connaissent au même moment en Afrique du Nord ou en Asie; dans une certaine mesure, ils rencontrent le même genre de difficultés avec les patois pyrénéens, provençaux, savoyards ou bretons par exemple.

En fait, c'est l'ensemble des stratégies missionnaires des différents ordres et des congrégations à l'intérieur de la France qui se trouve «importé » en Nouvelle-France. Or, cette importation, qui se fait systématique après 1632,

7. Comme l'écrit Jérôme Lalemant:

Je ne puis après tout désespérer. La primitive Eglise estoit remplie de bannis, de gens faits esclaves, de condamnez aux feux, aux roues, aux mines, aux escuries publiques; et Dieu a tiré de ces bassesses les tiares et les mitres, les sceptres et les couronnes qui ne trouveront leur affermissement solide que dans l'establissement du royaume de Jésus-Christ. Dieu vueille donner la pensée et le zèle aux princes chrestiens de l'establir en ce nouveau monde » (Relation de 1647, dans Campeau 1994, 754).

Voir aussi les références aux apôtres et à la vie apostolique, entre autres dans Campeau $1987,119,127,148,326,341,344,346,403,625,782,788,793$; 1989, 94$95,309,313,371,379,384,394,414,415,716,726,741,751,779$; 1990, 402, 509, 536; 1992, 83, 200, 216, 218, 650, 677 ; 1994, 75, 131, 371, 394, 396, 469, 471, 497, 554, 560, 598, 600, 609, 615, 754 ; 1996, 328, 346, 356, 837, 864, 897.

8. J. de Brébeuf, Relation de Huronie, 1636, dans Campeau 1987, 323 ; J. Lalemant, Relation de Huronie, 1641, dans Campeau 1990, 186. Voir aussi P. Biard, Relation de la Nouvelle-France, 1616, dans Campeau 1967, 538; C. Lalemant, Lettre à Jérôme Lalemant, 1627, dans Campeau 1979, 146, 154 ; M. Vitelleschi, Lettre à Jean de Brébeuf, 1630, dans Campeau 1979, 230-231 ; F. Le Mercier, Relation de Huronie, 1637, dans Campeau 1987, 768 ; etc. 
ne s'accomplit pas sans péril. Car avant même de débarquer sur le sol américain, les hommes et les femmes qui se font missionnaires, s'attendent à découvrir les mêmes défauts qu'ils ont l'habitude de combattre dans leur pays d'origine. Sur place, ils ne cessent de reconnaître ces défauts dans les comportements amérindiens qu'ils condamnent aussi violemment qu'en France. Pour eux, en effet, les mêmes démons sont à l'œuvre, et des deux côtés de l'Atlantique, les gens qu'ils séduisent sont de la même étoffe. Bref, nos missionnaires ne peuvent s'empêcher de reconnaître avant que de connaître.

On a souvent vanté les qualités d'observation des jésuites canadiens, qui nous valent aujourd'hui de précieuses sources ethnographiques et qui servent de fondement à l'histoire canadienne. Or, ce double souci de faire l'état des lieux et de sonder le sentiment religieux des peuples qu'ils rencontrent est celui de tous les missionnaires, aussi bien à l'intérieur de la France qu'à l'extérieur. Aussi, quand les missionnaires du Canada, religieux ou laïcs, examinent assidûment les mœurs et coutumes des Amérindiens, ils n'agissent alors pas autrement que ne le font leurs confrères des Antilles, du Moyen-Orient et d'Asie ou ceux des provinces françaises. Les relations de missions intérieures et lointaines montrent, en effet, que tous les agents convertisseurs suivent la pratique qu'avait si bien recommandée François Xavier, le grand modèle missionnaire, de "s'enquérir dextrement des mœurs ", des «vices et vertus » majoritaires du pays à convertir. Pensons à un César de Bus réfléchissant à la situation religieuse d'Avignon ou à un Julien Maunoir considérant le cas de la Bretagne et de ses îles; pensons à un François de Sales ou encore à un Chérubin de Maurienne mettant au point son plan de conquête spirituelle de la Savoie: les missionnaires de l'intérieur se font les observateurs attentifs des peuples qu'ils cherchent à ramener dans le giron de l’Église. Si leur «grille de lecture» leur fait considérer les « vices » qu'ils stigmatisent comme les fruits de l'ignorance religieuse, ils n'en laissent pas moins de précieuses informations à caractère ethnographique qui, à l'instar des rapports missionnaires concernant les Amérindiens, peuvent servir aujourd'hui à l'histoire ethnographique et socioculturelle des régions qu'ils ont parcourues?.

9. François Xavier 1648, 187. Francois de Sales 1892-1964, XI, 168-171. Voir aussi le plan du Capucin Chérubin de Maurienne décrit par Charles de Genève dans Charles de Genève 1976, vol. 1, 84-86, 92 ; vol. 2, 117 ; vol. 3, 215, 326-329. 
C'est pourquoi le jésuite Jérôme Lalemant est un missionnaire tout aussi typique que ses prédécesseurs missionnaires, quand, en 1642, il écrit de sa lointaine Huronie: "C'est beaucoup avancé [sic] que de recognoistre son monde, d'entrer dans les esprits, de se faire à leur langue, à leurs coustumes, à leur façon de vie et, s'il est besoing, se faire barbare avec eux pour les gaigner à Jésus Christ. » (Relation de Huronie, 1642, dans Campeau 1990, 545) Son confrère François Le Mercier le répète onze ans plus tard: ".. il se faut accoustumer aux coustumes et aux façons de faire des peuples qu'on veut gagner, quand elles ne sont pas éloignées de la raison. (Relation de 1653, dans Campeau 1996, 598) L'idée est là, qui perdure parmi les agents de la conversion; elle date des premiers apôtres et se décline en quatre temps : reconnaître, trier, adopter, modeler. On vient repérer chez l'Autre, les coutumes - dont le terme, nous rappelle Antoine Furetière dans son dictionnaire, «vient de l'italien costumi, qui signifie bonnes mours» (1690, article "Coutume») —; puis, on choisit celles qui sont compatibles avec le christianisme et on les embrasse, "on s'accoustume aux coustumes et façons de faire» (ibid.). Mais attention, l'objectif n'est pas de devenir l'Autre en intégrant ses traditions, en "s'acculturant» comme on dit abusivement aujourd'hui. Au contraire, il s'agit d'adopter, parmi les habitudes de l'Autre, celles que déjà on reconnaît comme siennes, qu'on juge conciliables avec " la raison ", c'est-à-dire ici la religion eurochrétienne. Ces coutumes, une fois adoptées, on les réoriente, on les plie selon les principes chrétiens, bref, on les christianise. En se faisant "gentil parmi les gentils ", on s'infiltre dans le système de valeurs de l'Autre, et, par le dedans, on le remodèle à sa guise. Qualifions le processus de mimétisme christianisateur ou d'inculturation, le résultat visé est le même: amener l'Autre à devenir Soi.

C'est encore ce qu'exprime Paul Ragueneau en 1648, quand il souligne la nécessité de "se faire tout à tous " [1Co 9,22] dans le but de changer l'Autre. Or, cette tâche psychologique dépasse en difficulté les épreuves physiques qui attendent les missionnaires: patience, courage, persévérance, humilité, indulgence, désintéressement sont autant de vertus nécessaires aux missionnaires qui se font «Sauvages» parmi les «Sauvages ${ }^{10}{ }$. Autrement

10. P. Ragueneau, Relation de Huronie, 1648, dans Campeau 1994, 393-394 (voir 370). Voir aussi, par exemple, les observations du jésuite Biard dans Campeau 1967, 140151, 475-478, 511-512, 600, 611-614, celles des récollets rapportées par Chrestien Le Clercq 1691, vol. 1, 91-100, 134-139, 264-265, 285-287 ; voir aussi le rapport de Denis Jamet, de 1615 dans Jouve 1915, 58-68. Voir, pour la France, Dompnier 1996, 156-161. 
dit, se faire tout à tous est une façon pour le missionnaire de gagner son ciel et de le faire gagner à ces Autres, tous ces Autres, qu'il entend convertir. Et, rappelons-le, cette aptitude des missionnaires à s'ajuster aux us et coutumes amérindiennes afin de les remodeler en les christianisant s'inscrit tout à fait dans la grande entreprise de normalisation des mœurs qui est alors en cours en Europe et qui fonde le mouvement de tridentinisation. Aussi, quand ils débarquent en Nouvelle-France, il leur est tout naturel de courir sus à ce qu'ils qualifient de "superstitions »; c'est tout bonnement ce qu'ils ont l'habitude de faire en France ${ }^{11}$.

C’est pourquoi il n'est pas étonnant que le jésuite François Du Péron tonne contre les coutumes, visiblement des «diableries », qu'il connaît bien en France et qu'il a l'impression de retrouver en Huronie: "Le 2 mars [1639] et les aultres jours ensuivans du carnaval, le diable est icy deschesné aussi bien qu'en France. Ce n'est que diablerie et masquarade en ce tempslà par tout le pays des Hurons. Cela a débauché deux ou trois de nos chrestiens et refroidy pleusieurs aultres qui se disposoient au baptesme ${ }^{12}$.» Et le jésuite de faire, dans l'urgence et la stupéfaction, une longue liste, qui reflète à la fois la qualité des observations ethnographiques et la mentalité obsidionale des missionnaires.

On le sait, les missionnaires s'intéressent de près aux croyances amérindiennes. Grâce à eux, d'ailleurs, qui ont contribué à fixer par écrit la tradition orale, nous connaissons les mythes des «Sauvages ». Ils n'ont de cesse, cependant, de reconnaître des traces de la révélation chrétienne - des traces du Déluge, des traces de l'incarnation du Christ — dans les récits fondateurs qu'ils rapportent tout en les jugeant absurdes, obscurcis par des siècles

11. Verjus 1666, 230 ; voir aussi 143, 166-168, 179, 185-192, 196-198, 215-216, 224, 226 et passim. Boschet 1834, 72-79, 111, 104-105, 164-166, 345. Charles de Genève 1976, vol. 3, 197-198; voir aussi 172, 173, 180, 236-238.

12. F. Dupéron, Lettre à son frère Joseph-Imbert Dupéron, 27 avril 1639, dans Campeau 1989, 222-225. Jérôme Lalemant décrit très longuement une séance de guérison qu'il assimile à une fête ou à un carnaval et où se produit :

... une manie générale de tous ceux du bourg qui, excepté peut-estre quelques vieillards, se mettent à courir partout où a passé la malade, mataschiez ou barbouillez à leur mode, avec des déformitez espouvantables de visage, à l'envy les uns des autres, faisant partout un tintamarre et des extravagances telles que, pour les exprimer et les mieux donner à entendre, je ne sçay si je les dois comparer ou à nos mascarades les plus extravagants dont on ait ouy parler, ou aux baccantes des anciens, ou plustost aux furies d'enfer. (Relation de Huronie, 1638-1639, dans Campeau 1989, 429, 428-432) 
d'infidélité. Cette disposition d'esprit va habiter longtemps les missionnaires - même les plus chevronnés. Et longtemps, les coutumes et croyances des Amérindiens vont apparaître aux missionnaires comme des extravagances incompréhensibles, des enfantillages, des charlataneries, voire des "superstitions diaboliques », dont il est relativement facile de les défaire. Face à la religion catholique, seule véritable, seule «raisonnable », ces coutumes sont des obstacles qui ne peuvent tout simplement pas tenir, soutiennent en chœur les missionnaires, puisque les Amérindiens eux-mêmes finissent par admettre leur absurdité: «... [ils] reviennent facilement de ces folles superstitions quand on leur en fait voir la vanité, et qu'on les instruit des véritez de notre sainte Religion, qui portant avec elles l'onction dans le cœur, leur donne un goût bien plus doux et plus innocent que ne font tous

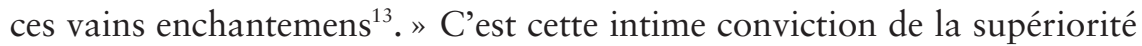
du christianisme qui sous-tend et guide l'effort des agents de conversion.

\subsection{Continuité des méthodes}

Dans l'ancienne comme dans la Nouvelle-France les méthodes convertisseuses sont rigoureusement les mêmes (je le démontre dans Deslandres 2003, 20-49, 87-196, 304-355). Elles consistent principalement, par la parole et par l'exemple, à émouvoir suffisamment afin d'amener les non-croyants à se convertir et les baptisés, à faire profession de foi ${ }^{14}$. C'est ce qui explique le caractère spectaculaire des missions. Comme la mission ad fidele en France,

13. P. Le Jeune, Relation de 1637, Campeau 1987, 562-563. Voir Marie de l'Incarnation 1971, 916 (dans la Lettre à son fils, 1670), 335 (Lettre à son fils, été 1647), 200 (Lettre à son fils, 1643), où l'ursuline rapporte avec un brin de condescendance :

Le Capitaine de cette Nation étoit un grand Sorcier, et l'homme du monde le plus superstitieux. Je lui écoutois soutenir la vertu de ses sorts et de ses superstitions, et peu après il vint trouver le Père contre qui il avoit disputé, lui apporta ses sorts et le tambour dont il se servoit dans ses enchantemens, et protesta de s'en vouloir jamais servir. Je vous envoye ce tambour afin que vous voyiez comme le Diable amuse et séduit ce pauvre peuple avec un instrument d'enfant; car vou sçaurez que cela sert à guérir les maladies, à deviner les choses à venir, et à faire de semblables choses extraordinaires.

14. Les signes de cette conversion sont le baptême pour les Amérindiens, la confession et la communion pour les chrétiens de France. Mais en France et en Nouvelle-France, le terme clé qui désigne globalement ce changement de cœur pour les missionnaires est la «conversion ". Voir au sujet des langues vernaculaires, par exemple, Boschet 1834 [1697], 41 et les relations de Pierre Biard (Campeau 1967, 538), Paul Le Jeune (Campeau $1979,146,154,230-231$, etc.). 
la mission amérindienne est conçue pour enflammer l'imagination des " païens » et créer l'ambiance adéquate pour la conversion. Or, les missionnaires sont des grands connaisseurs des cœurs ${ }^{15}$ qu'ils tentent d'apprivoiser par tous les moyens; des moyens déjà bien éprouvés dans l'ancienne France et qu'ils appliquent indifféremment aux paysans et aux «Sauvages », puisque, comme le souligne Paul Le Jeune, les âmes sont partout pareilles:

Pour l'esprit des sauvages, il est de bonne trempe. Je croy que les âmes sont toutes de mesme estoc et qu'elles ne diffèrent point substantiellement. C'est pourquoy ces barbares, ayans un corps bien fait et les organes bien rangez et bien disposez, leur esprit doit opérer avec facilité. La seule éducation et instruction leur manque. Leur âme est un sol très bon de sa nature, mais chargé de toutes les malices qu'une terre délaissée depuis la naissance du monde peut porter. Je compare volontiers nos sauvages avec quelques villageois, pource que les uns et les autres sont ordinairement sans instruction. Encore nos paysans sont-ils précipuez [avantagés] en ce point. Et néantmoins, je n'ay veu personne jusques icy de ceux qui sont venus en ces contrées qui ne confesse et qui n'advoue franchement que les sauvages ont plus d'esprit que nos paysans ordinaires ${ }^{16}$.

Avec les «exercices de la mission » (catéchisme, prédication, confession), les méthodes de conversion sont nombreuses, bien rodées, efficaces et vont des prières collectives aux processions, des chants didactiques aux pièces de théâtre, des images peintes aux œuvres de miséricorde (les visites des foyers, l'apaisement et la résolution des conflits, les œuvres charitables, les soins aux malades et aux prisonniers ${ }^{17}$ ). La venue des missionnaires est l'occa-

15. Le jésuite breton Julien Maunoir est à ce titre exemplaire : "Il connoissoit très-bien le cœur, et il en savoit le chemin. Tout ce qu'il disoit, alloit là ; et il sembloit qu'il remuât les passions comme il vouloit. Aussi le plus grand de ses talens étoit de toucher » Boschet 1834 [1697], 246, voir 43-44. «Le fruit que le Père prétendoit tirer de cette procession, c'étoit de frapper le peuple par des spectacles, et de lui faire entrer par les sens jusque bien avant dans le cœur l'amour de notre Seigneur, le souvenir de ses souffrances, la reconnoissance de ses bienfaits, la haine et la fuite du péché, l'esprit de pénitence, et un attachement inviolable au service de Jésus crucifié » (Boschet 1834 [1697], 280).

16. P. Le Jeune, Relation de 1634, dans Campeau 1979, 596. Voir Marie de l'Incarnation 1971, 809 (dans la Lettre à son fils, $1^{\text {er }}$ septembre 1668).

17. Ainsi :

Les pères ne furent pas plutôt arrivés à Quimper qu'ils s'employèrent à toutes sortes de bonnes œuvres. Le Père Maunoir alla à l'hôpital assister les malades. [...] Le Père alla ensuite visiter les prisonniers, consoler les malades de la ville et des faubourgs, porter des aumônes aux pauvres honteux, fortifier les personnes qui étoient 
sion de présenter, au moyen de techniques audiovisuelles sophistiquées, une religion sinon triomphante, du moins impressionnante. Il s'agit "d'imprimer quelques conceptions de la grandeur et vérité du christianisme ${ }^{18}$ ", en imprimant «dans des cœurs si bien préparés une crainte salutaire des jugemens de Dieu, qui les rende dociles aux mouvemens de la grâce » divine et aux instructions du missionnaire (Boschet 1834 [1697], 76-77, voir aussi 211, 280).

Or, comme ils jugent que les Amérindiens sont à peu près du même niveau intellectuel que les paysans français, les missionnaires vont utiliser le même genre de catéchisme et les mêmes moyens «audiovisuels » des deux côtés de l'Atlantique. Nous savons ainsi que Jean de Brébeuf et ses confrères de Huronie emploient le catéchisme de l'Espagnol Jacques Ledesma qu'ils traduisent en Huron. Cette Dottrina christiana breve per insegnare in pochi giorni per interrogationi a modo dialogo fra il mosstro et disceplo, composée vers 1573, est un petit in-octavo bien pratique d'une trentaine de pages, utilisé dans les missions intérieures et extérieures, dans lequel la doctrine est sommairement expliquée et où les questions et surtout les réponses sont d'une extrême simplicitée ${ }^{19}$. C'est une doctrine simple et éloquemment enseignée, mais c'est aussi une doctrine immuable: où qu'elles soient énoncées, ce sont toujours les mêmes idées qui sont mises de l'avant. Ce que les missionnaires disent à Québec est répété, point par point, par les missionnaires de Huronie ou d'Iroquoisie. Pas de place à la nouveauté ni à la spéculation intellectuelle ni surtout à la contestation — qui constituent l'apanage des

dans l'affliction, assister les maisons religieuses, et porter les épouses de JésusChrist à la perfection de leur état. Il s'adressoit aux magistrats pour les engager à remédier sans scandale aux désordres secrets, et avec éclat aux désordres publics : il prêchoit dans les paroisses, et inspiroit partout la fréquentation des sacremens, la charité envers les pauvres, l'union et la bonne intelligence des familles, la tempérance et la sobriété, et tout cela réveilloit et maintenoit l'ancienne ferveur. (Boschet 1834, 131-132)

Voir Boschet 1834, 260-289 ; Campeau 1967, 142, 218, 244, 511, 513 ; voir aussi, par exemple, 1979, 130, 134, 152, 154, 306, 317-318, 348, 384-386, 400-401, 447449, 468-472, 474, 506, 509, 516, 524-525, 529-530, 542-548, 559-563, 738, 776, etc. John O’Malley a décrit cette activité multiforme des jésuites (1995, 91-133, 165-199).

18. Biard, Lettre à Christophe Baltazar, provincial, 1612, dans Campeau 1967, 249.

19. Les citations sont de Paul Le Jeune, Relation de 1635, dans Campeau 1979, 447-448, voir aussi Relation de 1637, dans Campeau 1987, 111-112, 588. L'exposition de la doctrine par Jacques Ledesma suit le plan de la Somme du jésuite Pierre Canisius (Dhotel 1967, 99-100). 
protestants et des libertins. Il n'y a qu'une vérité, la catholique. Et cette cohésion doctrinale est alors comprise comme un puissant moyen de conversion. Jean de Brébeuf le note avec satisfaction:

... la conformité de tous les poincts de la doctrine chrestienne leur plaist merveilleusement : "Car, disent-ils, vous parlez conformément et tousjours consécutivement à ce que vous ave[z] dit; vous n'extravaguez point; vous ne dites rien hors de propos; mais nous autres, nous parlons à l'étourdy, sans sçavoir ce que nous disont». C'est le propre de la fausseté de s'embarasser dans une infinité de contradictions ${ }^{20}$.

Les missionnaires savent émouvoir - ils sont passés maîtres dans ce que j'appelle une douche écossaise d'émotions, c'est-à-dire qu'ils alternent savamment terreur et sécurisation. Usant de cette "pastorale de la peur» qu'a décrite Jean Delumeau, ils insistent particulièrement sur le jugement dernier et sur les peines de l'enfer ${ }^{21}$. Puis, ils amènent leurs auditeurs à un apogée émotionnel et, après les avoir profondément remués, ils changent le ton de leur discours et se font rassurants, invitant les missionnés à rejoindre la sécurité du christianisme tridentin ${ }^{22}$. À cette fin, les missionnaires utilisent toutes les occasions de la vie quotidienne et tout ce qui peut relever de l'extraordinaire: plus particulièrement, ils insistent sur la somptuosité des cérémonies, et plongent volontiers dans l'extraordinaire, quand comme les apôtres ils se fondent sur les guérisons miraculeuses, les prophéties et le martyr pour diffuser le message chrétien. Idéalement, l'usage de toutes ces techniques doit recevoir le soutien sans équivoque des autorités temporelles ${ }^{23}$.

20. Jean de Brébeuf, Relation de Huronie, 1636, dans Campeau 1987, 311.

21. Delumeau 1978, 23, 27, 45, 203, 216, 394 ; voir 1983, 369-627. Voir aussi Minois 1991, 260-261.

22. Deslandres 2003, 351s. Voir cette problématique développée par Dompnier (1983, 263).

23. Par exemple, Biard (dans Campeau 1967, 140-141, 244, 544, 546-547) et le séculier Michel Le Nobletz qui avise le jésuite Maunoir :

Il lui dit ensuite qu'il auroit à combattre l'ignorance et l'amour du plaisir ; qu'outre les moyens ordinaires de les détruire, qui sont les catéchismes, les prédications, les confessions, il lui conseilloit d'employer les chansons spirituelles, exprimant en vers bas-breton les dogmes et les maximes de l'évangile, et les faisant chanter sur des airs agréables et aisés à retenir. Les Calvinistes, lui dit-il, ont traduit les psaumes en vers français pour inspirer leur hérésie : servez-vous des chansons spirituelles pour inspirer au peuple la foi, l'espérance et la charité. Vous tirerez encore là un autre avantage; vous abolirez peu à peu l'usage des chansons deshonnestes. (Boschet $1834,70)$ 


\section{Des convertisseurs inconvertibles}

En évoquant le non-chrétien ou le pas tout à fait bon chrétien, en décrivant les traits qui les caractérisent et les aléas de sa mission, le missionnaire parle surtout de lui-même, de ses rêves, de ses projets et du modèle selon lequel il se construit et dont il est à la fois le propagateur, le garant et l'ardent défenseur. C'est pourquoi le nouveau, l'inconnu, n'a pu être vécu, analysé, compris par les missionnaires en termes de transformations personnelles profondes.

D’une façon générale, les missionnaires se sont souvent fait les hardis défenseurs des cultures qu'ils approchaient. Franciscains de NouvelleEspagne, jésuites de Chine, du Paraguay, du Canada ou de la France se sont évertués à trier les éléments culturels compatibles et incompatibles avec le christianisme. Leur choix, qu'on a voulu voir ethnographique avant la lettre, était tributaire de la civilisation eurochrétienne dont ils étaient issus. S'ils ont parfois cherché à préserver les modes de vie autochtones renouvelés par l'idéal chrétien, ils ne se sont guère rendu compte que ce renouvellement était dicté, le plus souvent, par les préceptes moraux et intellectuels propres à leur nation et à leur continent $t^{24}$.

Or les sources le démontrent: les missionnaires n'étaient pas venus, en ces terres de mission, pour changer. Ils étaient ce qu'on pourrait appeler «les incorruptibles de la foi », des "inconvertibles ${ }^{25}$ ", des êtres qui, trempés dans l'airain de la controverse, n'accepteraient jamais de se laisser convaincre par les idées religieuses de l'Autre.

Rappelons par exemple que Biard est un des controversistes réputés de son temps, que Le Jeune est un ancien calviniste (un converti donc plus catholique que le pape), que Brébeuf a résisté efficacement aux assauts propagandistes des réformés français. Rappelons que pour eux, la religion de l'Autre est considérée comme une erreur, une erreur malheureuse qu'il faut

24. Un tel ethnocentrisme n'est pas bien sûr l'apanage des seuls Européens. Combien de fois, en effet, les relations de mission ne rapportent-elles pas, avec un brin d'agacement et beaucoup d'ironie, la fierté évidente que montrent les autochtones à l'égard de leurs propres valeurs et de leurs traditions ? Ces derniers, en effet, cherchent souvent à convertir les missionnaires à leurs façons de faire et à leurs croyances. Pensons par exemple, à cet Amérindien montagnais qui enjoint fortement les Jésuites de NouvelleFrance de croire à la puissance des rêves car : "tout ainsi qu'il nous croyoit quand nous luy disions quelque chose, ou que nous luy monstrions quelque image, de mesme nous luy devions croire quand il nous disoit quelque chose propre de sa nation" (Paul Le Jeune, Relation de 1633, dans Campeau 1979, 436).

25. Notons que le terme "inconvertible» est employé au XVII ${ }^{\mathrm{e}}$ siècle, comme le montre Audisio 1996, 397. 
corriger. Aussi, oui, ces missionnaires qui se considèrent comme les apôtres de la Primitive Église, se font gentils parmi les gentils, adoptent les raquettes, le canot et le langage de l'Autre, mais ils ne changent pas fondamentalement leurs façons de considérer la vie et la mort, la civilisation et la barbarie, la liberté humaine.

Dans ce processus de la rencontre où l'on aurait pu croire que des échanges puissent se produire, les missionnaires sont là pour donner, pas pour recevoir des Amérindiens. Ils ne sont pas prêts à recevoir quelque chose qui pourrait les faire douter du bien-fondé de leur présence, qui pourrait les remettre en question. Ils sont alors convaincus qu'il est non seulement juste, nécessaire, mais obligatoire de propager la doctrine chrétienne et d'ainsi "sauver le monde".

Comme la damnation des hommes n'est en somme qu'une affaire de mauvaise éducation — d' " ignorance » de la foi — ceux qui sont «savants " en matière de la foi se sentent responsables de la perdition générale et tenus de rétablir la situation ${ }^{26}$. Il leur faut donc agir, promptement, efficacement, sinon ils devront en répondre devant Dieu; ils sont convaincus que leur propre salut passe par celui des autres. Dans cette perspective, il semble tout aussi juste de forcer les récalcitrants à se "sauver » malgré eux, c'est-à-dire de les « réduire », les re-conduire au christianisme, au besoin par la force.

\section{L'impossible adhésion}

Toutes ces constatations nous mènent à récuser l'emploi du terme acculturation qu'emploient de nombreux chercheurs pour rendre compte du phénomène missionnaire. En effet, ce terme, depuis son apparition en 1880, a subi un glissement sémantique révélateur. En 1936, les anthropologues le définissaient comme l'ensemble des mécanismes qui régissaient la rencontre de deux ou de plusieurs cultures ${ }^{27}$. Mais le terme a été récupéré par diverses disciplines, avec beaucoup de fruits ${ }^{28}$, puis il s'est inscrit dans la langue cou-

26. Par exemple, côté canadien, Pierre Biard, Relation de 1616, dans Campeau 1967, 614. Jean de Brébeuf, Relation de Huronie, 1635, dans Campeau 1987, 107, 115. Charles Lalemant, Lettre à son frère Jérôme Lalemant, $1^{\text {er }}$ août 1626, dans Campeau 1979, 151. Testament de Jean de Brébeuf, Relation de Huronie, 1637-1638, dans Campeau 1989, 151. Côté français : Boschet 1834 [1697], 426-427; voir 441. Vincent de Paul 1960, 75, 44-45, voir 70, note 18, 74, 77. Jean Eudes renchérit dans Eudes 1906, 57.

27. Redfield, Linton et Herskovits 1936, 149 ; Wachtel, 1974 ; Bastide 1998, 1-114c ; Baré, 1992, p. 2. 
rante. Cette inscription n'a pas toujours été heureuse; il n'y a qu'à ouvrir nos dictionnaires: ainsi parle-t-on aujourd'hui de "l'acculturation des Amériques » en voulant décrire le processus par lequel les Amérindiens ont assimilé les valeurs européennes; on évoque «l'acculturation d'un émigré » en voulant rendre compte de l'adaptation d'un individu à une culture étrangère avec laquelle il est en contact; ou alors on identifie le phénomène à l'adaptation à un milieu psychosocial.

Tous ces usages reflètent une réduction du sens; et souvent ils s'entachent d'une tournure d'esprit qui n'envisage la rencontre de deux ou de plusieurs cultures qu'en termes hiérarchiques, avec l'inévitable absorption de la culture dite «faible» ou « inférieure » par la culture «dominante » ou «supérieure». Tout l'aspect d'échanges, d'interpénétrations culturelles, qui faisait la richesse du concept d'acculturation, est ainsi perdu. C'est pourquoi nous devons manier prudemment ce concept et préciser que la " conversion » n'est pas l' " acculturation », que les missionnaires, c'est-à-dire les «agents convertisseurs », ne sont pas des agents «acculturants» et que chercher à intégrer l'Autre à un modèle socioreligieux n'est pas un projet d'acculturation socioreligieuse; user autrement du terme acculturation, dans le contexte de l'histoire de la rencontre franco-amérindienne du XVII ${ }^{e}$ siècle, signifierait que les missionnaires étaient prêts à changer le message chrétien, à se laisser gagner par la religion de l'Autre. Ce qui, à l'époque du jésuite Laforgue, n'était évidemment pas le cas.

\section{Références}

Audisio, G. (1996), Les Français d'hier, t. I: Des croyants. XV'-XIX siècle, Paris, Colin.

Authier, C. (1641), Lettre du $1^{\text {er }}$ octobre 1641 au secrétaire de la Congrégation de la Propagande de la Foi, dans Archives de la Congrégation de Propaganda Fide: S.C. Francia, vol. 1, folio 89r.

Axtell, J. (1992), «Imagining the Other: First Encounters in North America", dans Beyond 1492: Encounters in Colonial North America, Oxford, Oxford University Press, p. 25-74.

28. On pensera par exemple aux travaux de l'anthropologue Nathan Wachtel, dont La vision des vaincus paru en 1971 et à ceux de l'historien Serge Gruzinski (1999; 2004). Ces deux collègues de l'École des Hautes Études en Sciences Sociales ont aussi en 2001 produit ensemble Le Nouveau Monde, Mondes nouveaux. L'expérience américaine. Voir aussi Berry 1989; Camilleri et Cohen-Emerique 1989, 29. 
BARÉ, J.-F. (1992²) [1991], "Acculturation », dans P. Bonté et M. IZARD, dir., Dictionnaire de l'ethnologie et de l'anthropologie, Paris, Presses universitaires de France.

BAstide, R. (1998) [1968], "Acculturation ", dans Encyclopédie Universalis, vol. 1, p. 114s.

BERRY, J.W. (1989), "Acculturation et adaptation psychologique», dans J. Retschitzki, M. Bossel-Lagos et P.R. Dasen, dir., La recherche interculturelle, Paris, L'Harmattan, t. I, p. 135-145.

Berthelot du Chesnay, C. (1967), Les missions de saint Jean Eudes. Contribution à l'histoire des missions en France au XVII siècle, Paris, Procure des Eudistes.

Boschet, R.P. (1834) [1697], Le parfait missionnaire ou la vie du R.P. Julien Maunoir, de la Compagnie de Jésus, missionnaire en Bretagne, Paris, Anisson.

Bourdoise, A. (1660), L'idée d'un bon ecclésiastique ou les sentences chrestiennes ou clericales, Paris, Bresche.

CAmilleri, C. et M. Cohen-Emerique (1989), Chocs de cultures : concepts et enjeux pratiques de l'interculturel, Paris, L'Harmattan.

Campeau, L., éd. (1967), La première mission d'Acadie (1602-1616), Rome / Québec, Monumenta Historica Societatis Iesu / Presses de l'Université Laval (Monumenta Novæ Franciæ 1).

- (1979), Établissement à Québec (1616-1634), Rome / Québec, Monumenta Historica Societatis Iesu / Presses de l'Université Laval (Monumenta Novæ Franciæ 2).

- (1987), Fondation de la mission huronne (1635-1637), Rome / Québec, Monumenta Historica Societatis Iesu / Presses de l'Université Laval (Monumenta Novæ Franciæ 3).

- (1989), Les grandes épreuves (1638-1640), Rome / Montréal, Institutum Historicum Societatis Iesu / Bellarmin (Monumenta Novæ Franciæ 4).

- (1990), La bonne nouvelle reçue (1641-1643), Rome / Montréal, Institutum Historicum Societatis Iesu / Bellarmin (Monumenta Novæ Franciæ 5).

- (1992), Recherche de la paix (1644-1646), Rome / Montréal, Institutum Historicum Societatis Iesu / Bellarmin (Monumenta Novæ Franciæ 6).

- (1994), Le témoignage du sang (1647-1650), Rome / Montréal, Institutum Historicum Societatis Iesu / Bellarmin (Monumenta Novæ Franciæ 7).

- (1996), Au bord de la ruine (1651-1656), Rome / Montréal, Institutum Historicum Societatis Iesu / Bellarmin (Monumenta Novæ Franciæ 8). 
Cañizares-esguerra, J. (2002), How to Write History of the New World: Histories, Epistemologies, and Identities in the Eighteenth-Century Atlantic World, Stanford, Stanford University Press.

Charles de Genève (1976) [1643], Les trophées sacrés ou missions des capucins en Savoie, dans l'Ain, la Suisse romande et la vallée d'Aoste, à la fin $d u X V I^{e}$ et au XVII siècles / édité par F. Tisserand, Lausanne, Société d'histoire de la Suisse romande, $3^{\mathrm{e}}$ série, 3 vol.

Clendinnen, I. (1987), "Franciscan Missionaries in Sixteenth-Century Mexico ", dans J. Obelkevich, L. Roper et R. Samuel, dir., Disciplines of Faith: Studies in Religion, Politics and Patriarchy, Londres / New York, Routledge, p. 229-245.

— (1990), "Ways to the Sacred: Reconstructing "Religion" in SixteenthCentury Mexico », History and Anthropology, 5, p. 105-141.

Daniel-Rops, H. (1966), Histoire de l'Église du Christ, vol. 6 : La réforme catholique, Paris, Gründ.

Descourveaux, P. (1714), La vie de monsieur Bourdoise, premier prestre de la communauté de S. Nicolas du Chardonnet, Paris, Fournier.

Delacroix, S., dir. (1957), Histoire universelle des missions catholiques d'après la conception originale de J.L. Françoisprimo, vol. 2: Les missions modernes, Paris, Gründ.

Delumeau, J. (1978), La peur en Occident. XIV'-XVII siècles. Une cité assiégée, Paris, Fayard.

- (1981), Un chemin d'histoire. Chrétienté et christianisation, Paris, Fayard.

- (1983), Le péché et la peur. La culpabilisation en Occident. XIII ${ }^{e}$ XVIII ${ }^{e}$ siècles, Paris, Fayard.

- (1989), Rassurer et protéger. Le sentiment de sécurité dans l'Occident d'autrefois, Paris, Fayard.

Delumeau, J. et M. CotTret (1996) [1971], Le Catholicisme entre Luther et Voltaire, Paris, Presses universitaires de France (Nouvelle Clio).

Deslandres, D. (1997a), "Les missions françaises intérieures et lointaines, esquisse géo-historique ", Mélanges de l'École française de Rome, 109/2, p. 505-538.

- (1997b), "L'Amérique ibérique », dans J.-M. MAYeur et al., dir., Histoire du christianisme, vol. 9: L'âge de raison (1620-1750), Paris, Desclée, p. 618-664.

- (2003), Croire et faire croire. Les missions françaises an $17^{\circ}$ siècle, Paris, Fayard. 
Dhотец, J.-C. (1967), Les origines du catéchisme moderne d'après les premiers manuels imprimés en France, Paris, Aubier.

Dompnier, B. (1983), « Pastorale de la peur et pastorale de la séduction. La méthode de conversion des missionnaires capucins ", dans La conversion au XVII siècle, Marseille, Centre Méridional de Rencontres sur le XVII ${ }^{\mathrm{e}}$ siècle.

- (1996), "La compagnie de Jésus et la mission de l'intérieur ", dans L. Giard et L. de Vaucelles, Les Jésuites à l'âge baroque, 1540-1640, Grenoble, Millon, p. 156-161.

Duverger, C. (1987), La conversion des Indiens de la Nouvelle Espagne, Paris, Seuil.

Elliott, J.H. (I970), The Old World and the New, 1492-1650, Cambridge, Cambridge University Press, p. 15-16.

- (1972), The Discovery of Natural Man and the Discovery of Man, London, Oxford University Press.

- (1976), «Renaissance Europe and America: A Blunted Impact?», dans F. Chiappelli, dir., First Images of America: The Impact of the New World on the Old, Berkeley, Berkeley University Press, p. 11-23.

Eudes, J. (1906) [1648], CEuvres complètes du Vénérable Jean Eudes, Paris, Beauchesne, t. II.

Farriss, N.M. (1984), Maya Society under Colonial Rule: The Collective Enterprise of Survival, Princeton, Princeton University Press.

François de Sales (1892-1964) [1595], Lettre au duc Charles-Emmanuel $I^{e r} 29$ déc. 1595, dans CEuvres, Annecy, Monastère de la Visitation, t. XI, p. $168-171$.

François Xavier (1648) [1549], Lettre à Gaspard Barzé [début avril 1549], dans C. Borromée, Instructions aux Confesseurs, Toulouse, Colomiez.

FuretiÈre, A. (1690), Le dictionnaire universel, La Haye / Rotterdam, Arnout \& Reiner Leers.

Gliozzi, G. (2000) [1992], Adam et le nouveau monde. La naissance de l'anthropologie comme idéologie coloniale: des généalogies bibliques aux théories raciales (1500-1700) / trad. par A. Estève et. P. Gabellone, Lecques, Théétète.

Grafton, A., A. Shelford et N. Siraisi (1992), New Worlds, Ancient Texts: The Power of Tradition and the Shock of Discovery, Cambridge, Cambridge University Press, p. 28-58, 116. 
Griffiths, N. et F. Cervantes, dir. (1999), Spiritual Encounters: Interactions between Christianity and Native Religions in Colonial America, Birmingham, University of Birmingham Press.

Gruzinski, S. (1988), La colonisation de l'imaginaire, Paris, Gallimard.

- (1999), La pensée métisse, Paris, Fayard.

- (2004), Les quatre parties du monde. Histoire d'une mondialisation, Paris, La Martinière.

Grunzinski, S. et N. Wachtel, dir. (2001), Le Nouveau Monde, Mondes Nouveaux. L'expérience américaine, Paris, École des Hautes Études en Sciences Sociales.

JAMET, D. (1915) [1615], Rapport de 1615, résumé et publié dans O.-M. Jouve, Les Franciscains et le Canada. L'établissement de la foi 16151629, Québec, Couvent des S. Stigmates, p. 58-68.

Le ClercQ, C. (1691), Premier Etablissement de la Foy dans la NouvelleFrance, Paris, Auroy, 2 vol.

MacCormack, S. (1991), Religion in the Andes: Vision and Imagination in Early Colonial Peru, Princeton, Princeton University Press.

Marie de L'InCARnation (1971) [1626-1671], Correspondance [de 1626 à 1671] / édité par G.-M. Oury, Solesmes, Abbaye St-Pierre.

Maunoir, J. (1998) [г650], Miracles et sabbats. Journal du père Maunoir. Missions en Bretagne. 1631-1650 / édité par É. Lebel, Paris, Éd. de Paris (Essais et documents).

Minois, G. (1991), Histoire des enfers, Paris, Fayard.

O'Gorman, E. (1961), The Invention of America: An Inquiry into the Historical Nature of the New World and the Meaning of its History, Bloomington, Indiana University Press.

O'Malley, J. (1995), The First Jesuits, Cambridge / London, Harvard University Press.

Pagden, A. (1982), The Fall of Natural Man: The American Indian and the Origins of Comparative Ethnology, Cambridge, Cambridge University Press.

Redfield, R., R. Linton et M.J. Herskovits (1936), « Memorandum for the Study of Acculturation ", American Anthropologist, 38/1, p. 149152.

Rogier, L.-J., R. Aubert et M.D. Knowles, dir. (1968), Nouvelle histoire de l'Église, vol. 3 : Réforme et Contre-Réforme, Paris, Seuil. 
Schmidt, B. (2002), Innocence Abroad: The Dutch Imagination and the New World, 1570-1670, Cambridge, Cambridge University Press.

Seumois, A.V. (1952), Introduction à la missiologie, Suisse, SchöneckBeckenried (Nouvelle revue de science missionnaire. Supplementa 3).

Todorov, T. (1982), La conquête de l'Amérique. La question de l'Autre, Paris, Seuil.

- (1989), Nous et les Autres. La réflexion française sur la diversité humaine, Paris, Seuil.

Vincent de Paul (1960), Entretiens spirituels aux missionnaires. Textes réunis et présentés par André Dodin, Paris, Seuil.

Verjus, A. [pseudonyme: Antoine de Saint-André] (1666), La Vie de monsieur Le Nobletz, prestre et missionnaire, Paris, François Muguet.

Wachtel, N. (1971), La vision des vaincus. Les Indiens du Pérou devant la conquête espagnole, Paris, Gallimard.

(1974), «L'acculturation », dans J. Le Goff et P. Nora, dir., Faire de l'histoire, t. I: Nouveaux problèmes, Paris, Gallimard (Folio. Histoire).

Zupanov, I. (1991), Writing and Acting Cultures: The Jesuit Experiment in $17^{\text {th }}$ Century South India, thèse de Ph. D., University of California.

\section{Résumé}

La rencontre des croyances franco-amérindiennes du XVII siècle doit être replacée dans le contexte plus global des missions de cette époque. On voit alors se développer simultanément les missions à l'intérieur de la France, auprès des catholiques tièdes et des protestants, et les missions à l'extérieur de la métropole, auprès entre autres des Amérindiens de Nouvelle-France. Or, l'analyse révèle que, dans son ensemble, la mission française au XviI ${ }^{\mathrm{e}}$ siècle fut loin d'être un lieu d'innovation. L'altérité, en effet, a eu un impact quasi nul sur les mentalités et les méthodes missionnaires. D’une part, les missionnés étaient perçus essentiellement comme des «ignorants » de "ce qui est nécessaire à salut ", dont il serait facile de circonvenir les superstitions; d'autre part, c'est l'ensemble des stratégies missionnaires des différents ordres et des congrégations à l'intérieur de la France qui se trouva «importé » en Nouvelle-France. Dans ce processus, la rencontre de l'Autre se fait à sens unique, les missionnaires étant là pour donner et non pour recevoir des missionnés quelque chose qui pourrait les remettre en question ou susciter leur adhésion à la religion de l'Autre. 


\begin{abstract}
The Franco-Amerindian encounter of beliefs must be replaced in the global context of $17^{\text {th }}$ century missions, which see simultaneously the development of missions inside France, among Catholics and Protestants, and outside the country, among peoples like the Amerindians of New France. The analysis reveals that, as a whole, the French mission was far from being a place of innovation. Otherness, in effect, has had no impact on missionary spirit and methods. On the one hand, the "missionized" were essentially perceived as ignorant of what was necessary for salvation, peoples whose superstitions would be easily denied. On the other hand, it was as a whole that the missionary strategies of the diverse orders and congregations of France were imported in New France. In this process, the French encounter of the Other was only in one direction, the missionaries being there to give, not to receive anything from the Other that could question their legitimacy or cause their adhesion to the religion of the Other.
\end{abstract}

